\title{
Future Network Applications, Network Model, and Development Strategy
}

\author{
Jun DOU, Yu XIA, Xi CHEN \\ Sichuan Network Communication Technology Key Laboratory (SC-Netcom Lab) \\ Southwest Jiaotong University \\ Chengdu, P.R. China \\ Email: jdou@home.swjtu.edu.cn
}

\begin{abstract}
This paper focuses on important issues pertinent to future networks, i.e. future network application trend, Network Model, and NGN development strategies. The authors challenge the orthodox OSI/RM and Internet architectural representation with a point of view that it does not explain modern network operation satisfactorily and is unable to cope with service oriented trend in network computing. By re-clarifying out-of-band signaling concept in the context of networking, this paper provides an architecture representation of Internet and introduces an ONAS/RM (Open Network Application Service/Reference Model) for future networks. With regard to NGN development strategy, this paper promotes a "BSF-OES" (Backbone Substrate First, Outwards Expansion Second) strategy, in contrast to "add-on" approaches widely adopted in standards organizations and to those termed by layer-less architecture. Finally, a framework of SUPANET (Single-layer User-data switching Platform Architecture Network), developed at SC-Netcom Lab, is used to demonstrate feasibility of such strategy and how to cope with technical challenges, to protect investments, and to make smooth migration to future networks.
\end{abstract}

Keywords-Future Network; OSI/RM; ONAS/RM; BSF-OES; SUPANET

\section{INTRODUCTION}

World-wide application of Internet has made it a de facto standard of computer networks in the past. However, diversity in nature of network-application traffic, in network applications, in communication techniques, and in network attacks has put Internet to confront with ever-growing challenges, spanning from high-speed switching, through QoS provisioning, security, and mobility to service-oriented network applications. Having failed in solving these problems and in development of next generation Internet simply by replacing IPv4 with IPv6, more and more people have realized the need for "clean slate" network architectures [1] with different interpretations though.

Over the years, ever-growing security patches (to almost all layers and protocols) and enhancement in meeting application requirements have made Internet more and more complicated and less efficient. This is contradictory to high datarates up to $160 \mathrm{Gbps}$ per lambda in optic communications, which have imposed great challenges to packet switching techniques in electronic domain and exact simplification of switching platform of network nodes. The very low error-rate in optics $\left(10^{-12}\right)$ and in UTP $\left(10^{-11}\right)$ defies the necessity of having an independent data link layer for error-checking in backbone networks, nevertheless in access domain, high error rate (e.g. over wireless and telephone lines) still needs errorchecking in the data link layer. The conflict caused by diverse communication techniques is questioning the possibility of defining a unified layered structure as in OSI/RM.

Analysis has also shown that OSI/RM [2] and Internet model were designed with an idea that common network applications could all be included in the application layer of the network model to enable open-system interconnection. Therefore, no application-layer service and service interface have been defined in OSI/RM or Internet. Nevertheless, recent development in network computing has shown that lack of such service interface has blurred demarcation between real applications that utilize network to provide applicationoriented service, and the network application platform that provides open information exchange. This has made development of new network computing system difficult. For example, the OGSA (Open Grid System Architecture) [3] defined by OGF (Open Grid Forum) has to merge grid platform with application grids and resulted in a self-contained architecture, difficult to understand for network communities [4]. Cloud Computing [5], characterized by deliver services to customers via Internet, is another example of demanding a clear service boundary between cloud applications and the network that supports service delivery.

All these considerations have led to the research work presented in this paper related to following issues:

A. How should the OSI/RM be adapted to modern network computing requirements and be refined to describe network operation appropriately? Is it feasible to define a uniform network architecture for future networks with diverse communication techniques?

B. What strategy should we take in development of future networks? And

C. What architecture can we have for future backbone networks?

To answer these questions, this paper analyzes inadequacy of OSI/RM and provides a new representation of Internet

This paper has been supported by two consecutive grants from CNSF (with grant numbers 60372065 and 60773102 respectively). 
architecture, proposes a refined model for future networks, and promotes a development strategy called "BSF-OES" (Backbone Substrate First, Outwards Expansion Second) based on the framework of backbone network architecture called SUPA (Single-layer User-data switching Platform Architecture) [6].

\section{NEW ARCHITECTURAL REPRESENTATION OF INTERNET AND NETWORK MODEL}

OSI/RM [2] has been the orthodox reference model in dealing with network interconnection issues since 1980; and Internet architecture was later represented to conform to the model (except that three OSI upper layers are merged as the Internet application layer). OSI/RM assumed that common network applications, such as file transfer, World Wide Web, e-mail, and etc. can all be included in the Application Layer of the model. In other words, it was intended to define an interconnection model for open network-systems rather than a model for open application systems interconnection through an open network application platform.

The new trend of SOA (Service Oriented Architecture) in application systems is promoting systems package functionality as interoperable services [7], consequently it demands that computer networks provide a generic platform for distributed information exchange (related to data, control, and management). With ever-growing applications of diverse requirements over Internet, inclusion of all applications into the application layer becomes more and more difficult and impractical. Lack of network application service in OSI/RM has blurred the boundary between the real application system dealing with business-oriented services and network application layer. Indeed, multiple overlay-networks [8] like P2P [9], Grids [3], and cloud computing [5] built as an application layer functions have caused much confusion with regard to OSI/RM. Having analyzed architectural confusion of OGSA (Open Grid Service Architecture), reference [4] suggested that an open network application service interface should be defined between application grids and network application layer supporting required grid applications. This stresses the necessity of defining a network application-layer service boundary to separate the real application from the application layer in OSI/RM.

Another problem with OSI/RM was caused by the assumption that all the user-data and control and management information in subnetworks were handled over the same platform involving the network layer at most. This assumption contradicts with real operation of subnetworks in Internet, where routing protocols, SNMP, DNS, \& etc. are actually utilizing transport protocols. In other words, they should be better referred to as special types of application-layer protocol for network operation and administration.

However, such contradiction can easily be avoided by reclarification of the "out-of-band signaling" concept in the context of computer networks. "Out-of-band signaling" was originally introduced in communications to describe that control data is transmitted in different channel (represented by frequency band) from the one for user-data (i.e. transmission "band"). When this concept was lent into computer networks, no one had tried to clarify differences of connotation of the term "band" in two cases. Obviously, "band" in the context of networks has, in most cases, lost its original meaning - "frequency band" and is likely to stress the layered structure, protocol stack, or simply an information exchange platform, even if different frequency bands may be involved in some cases. With the re-clarified "out-of-band" signaling concept, we can redraw the layered structure of existing Internet as in Figure 1, where a user-data switching platform (U-platform) is separated from that for control and management information (S\&M-platform - Signaling \& Management-platform) involving different layers respectively.

As shown in Fig. 1, horizontally the traditional network is formed of a subnetwork and a resource network. With out-ofband signaling concept, the subnetwork is further divided into two interrelated platforms: the U-platform and the S\&Mplatform. The former involves three lower layers in OSI/RM; while the latter involves all the OSI layers up to the application layer. Therefore, components of S\&M-platform in relay systems form another type of end-system (let's call them Signaling \& Management End-Systems, S\&M-ES for short). To differentiate the S\&M-ES from the "End-system" defined in OSI/RM, which is now referred to as AO-ES (ApplicationOriented End-System) in resource networks.

Another difference between Fig. 1 and OSI/RM is that the resource network is now divided into two parts: the real applications and AO-ES (Application-Oriented End-System (i.e. network application service provider). With the architectural representation of Internet as in Fig. 1, special protocols, such as RIP, SNMP, BGP, DHCP, and DNS can easily be viewed as application protocols in routers since they utilize transport protocols and are uniquely identified by dedicated IP address and associated port number.

It should be stressed that the out-of-band signaling concept can be further extended to AO-ES considering that

A. Some application protocol like FTP [10] has been using two TCP connections: one for control and the other for real file transfer. Consequently, control information is exchanged in a platform other than the one for files.

B. In many cases, an entity in an AO-ES has to first find out the destination IP address via DNS in S\&M-platform of subnetworks, if an AO-ES only knows UNL (Universal Name Locator). Operations of ARP and DHCP are also examples of out-of-band signaling between $S \& M-E S$ and $A O-E S$. This, to some extent, has shown the universal applicability of out-of-band signaling.

C. The potential merit of adopting out-of-band signaling in $A O-E S$ is to reduce mutilayer overheads in U-platform, espeially for multimedia streams. As a result, layers in $A O-E S$ can also be reduced to enable loading segmented data streams into payload part of PDU of the substrate. 




Figure 1. Interpretation of Internet layered Architecture with a re-clarified out-of-band signaling concept

Another issue worthwhile rethinking is the soundness of making a sharp distinction between network address (IP in Internet) and the transport address (i.e. port number in Internet). As shown in figure 1, exchange of control and management information in a subnetwork is by and large done between application entities in S\&M-ESs, which has to be identified jointly by an IP address and a port number. For a connection-oriented protocol like RSVP [11], the hop-by-hop path negotiation involves application entities in routers, which needs both IP address (to identify network interface of a router) and port number (to identify application process). A potential gain with out-of-band signaling might be to merge existing network layer and transport layer into a single layer in S\&M-platform in the future. People might argue that it does not need port number for switching IP packets in current Internet. It is true but in the future, IP protocol is likely be degraded to identify physical address (physical port of a router or a host) during connection establishment, while in data transfer phase, switching of user-data over the U-platform, needs only connection Id, not IP address any more for connection oriented service. In this case, service negotiation (e.g. for QoS) has to involve application entities, which needs to be identified by port number of transport protocol.

Perhaps, the most prominent gain obtained from out-ofband signaling to provide connection-oriented service, is to simplify the U-platform. In fact, B-ISDN and MPLS have reduced the U-platform to a two-layer structure and we have been trying to build a single-layer U-platform [12].

Criticism to layered structure in current networks comes from wireless networks, where higher layer operations, such as routing decision making, demand cross-layer operation to access low layer information (e.g. error rate in physical channel or frame loss rate of data link layer). It seems to be a strong point against layering architecture. However, this can easily be avoided by relaxing the rigid regulation of layer-by- layer operation, for example, to permit bypassing a layer whenever necessary. This is just a matter of regulations.

In sum, the OSI/RM and current Internet architecture representation can be refined with the concept of out-of-band signaling to provide a better explanation to interrelationship among protocols in current Internet and a basis for future network development. More separately importantly, further architectural simplification can be done over two different platforms with the out-of-band signaling concept.

\section{NGI DEVELOPMENT STRATEGY}

As briefly mentioned in introduction, there are different development strategies towards NGN/NGI. The authors have taken a strategy called "BSF-OES" (Backbone Substrate First, Outwards Expansion Second) towards NGI [13]. This strategy is based on the vision of communication world where future backbone networks will be based on low error-rate DWDM with very high bit rates; while access networks will be based on diverse communications techniques possibly with high error-rates. Fig. 2 illustrates our vision for NGI development.

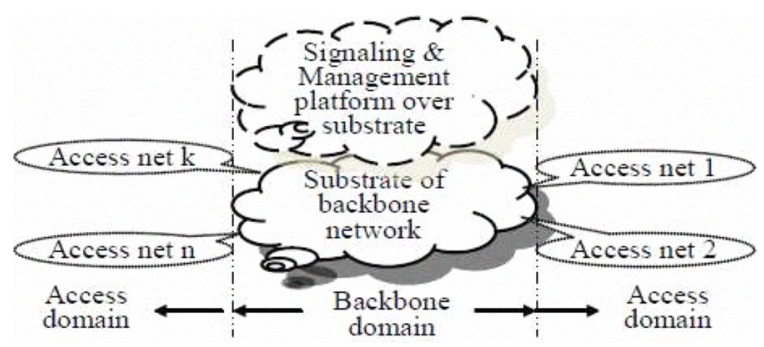

Figure 2. Our vision for future interconnected network environment with out-of-band signaling concept 
The substrate ${ }^{1}$ of the backbone network in Fig. 2 is the bottom stratum in subnetworks, which should provide reliable, secured, QoS insurable, high-speed encapsulating or "ferrying" service for user-data among access networks. It can also provide encapsulation service for signaling and management information in S\&M-platform. In view that DWDM is capable of providing transmission rate at order of $\mathrm{T}$ bps over a single fiber; it will surely be the most promising technique for building the substrate of backbone subnetwork. Here, the "Backbone Substrate First" implies first to focus on building a thin substrate to cope with high-speed switching problem with embedded QoS mechanism and security measures. "Outwards Expansion Second", on the other hand, implies that outwards expansion will be done only when backbone technologies are fledged and wherever applicable.

In view that Internet is the largest operational network up to date, with large amount of investment and operation experiences, interoperability with Internet and smooth migration from Internet must be considered in NGI development. For the very reason, in our first phase development of SUPA [6], we kept Internet protocol stack in the S\&M-platform of SUPANET to maintain interoperability between Internet and SUPANET.

\section{A NETWORK MODEL FOR FUTURE NETWORKS}

With regard to network modeling, there are two different approaches: layering and layer-less. OSI/RM and Internet model promote layering approach, with which functional modules are represented as interrelated layers. Layers except the Application layer all provide implicitly (in Internet) or explicitly a service to their adjacent layer above while behavior of peer entities in the same layer is regulated by the layer protocol.

Such layering architecture has been criticized for various reasons. One strong point against layering architecture is that the rigid regulation on layer-by-layer operation prevents higher layers from efficient cross-layer access to low layer information such as transmission error-rates. However, such convenience can be obtained by relaxing the rigid layer-by-layer operation rule should it be necessary. In this sense, it is no longer a layering or layerless issue, rather how can a layered structure be more flexible.

It is worth mentioning that some NGI architectures such as FARA [14] declared to be "layer-less", are at most partially layer-less since the most important part, i.e. the subnetwork is referred to as a substrate and excluded from their discussion $[14,15]$. In other words, these architectures focus on how to represent the modules of high-layer functions and how modules or sub-modules can be interrelated or invoked in a more flexible or convenient way for implementations, such as object-oriented or role-based programming.

\footnotetext{
${ }^{1}$ The meaning of "substrate" in computer networks is blurred. Here it used to describe the lowest stratum, which relays bit streams, frames, or packets across a subnetwork.
}

In view that the crux of future networks is to make a subnetwork a high-speed, high-efficient, QoS-insurable, secure, flow-controllable, congestion-controllable, traffic-engineering supported, and maintainable, we have been focusing our research efforts on subnetworks. Introduction of the out-of-band signaling enables separation of U-platform from S\&Mplatform and consequently reduces the U-platform to minimal, especially over optical fiber communications in backbone. With the analysis above, we have three considerations in developing an Open Network Application Service/Reference Model (ONAS/RM) for future networks as shown in Fig. 3:

A. Similar to the refined Internet model in Fig. 1, the new model should exclude the diverse network applications from the model and only those common functions supporting open interconnection for real application systems over network are left in the model. Therefore, a Network Application Service Interface (NASI) should be explicitly defined.

B. Similar to Fig. 1, two types of end-system, i.e. S\&M-End System (S\&M-ES) and Application-oriented End-System (AO-ES) are needed for subnetworks and for resource networks (i.e. the part formed of the End-systems defined in OSI/RM). Consequently, the Transport and network layers as in Fig. 1 should be merged into one if possible.

C. For efficiency, a compact substrate layer should be defined to switch bit-blocks (e.g. physical layer frames) carrying higher-layer PDUs both for U-platform and $S \& M$-platform among communication channels.

As shown in Fig. 3, ONAS/RM explicitly adopts the concept of out-of-band signaling; therefore,

A. Subnetworks as well as the AO-ESs are formed of two interrelated platforms, i.e. the U-platform and the $S \& M$ platform involving different protocol layers respectively.

B. A relay system is a device within which $N$ input-units and $N$ outpu-units are interconnected via the relay function (" $R$ " in Fig.3). The layer that the basic units are processed and relayed decides the layer of the substrate.

C. User data encapsulated in PDU of the substrate is directly switched to releant output port without need to know what they are (shown by bold line), while highlayer protocol data units of S\&M-platform encapsulated in PDU of the layer of substrate may either be taken out and handed to higher layers or indirctly swiched by the relay function to relevant output port as shown by dotted lines in Fig.3. 


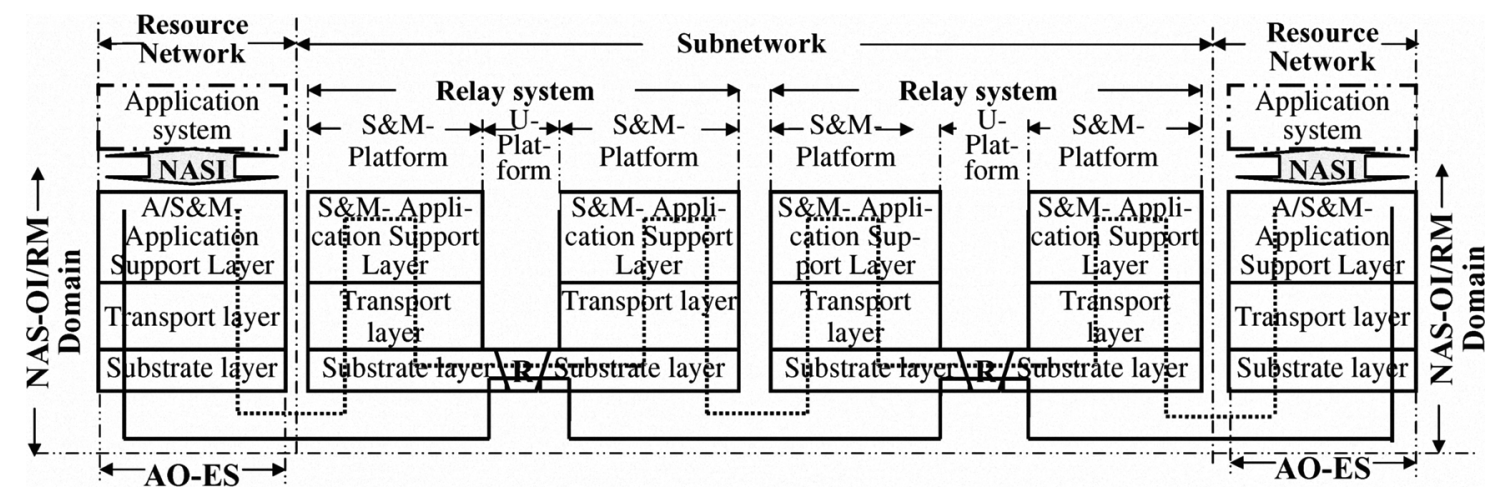

Figure 3. The ONAS/RM for Future Internet with Out-of-band signaling

\section{CAN WE HAVE A SINGLE-LAYER SUBSTRATE}

In view that many quantifiable QoS parameters (e.g. throughput, jitter, etc.) are essentially performance measure of transmission and switching, a switching platform with multiple layers for user-data can cause more non-deterministic characteristics in network performance and be hindrance to high-speed switching and $\mathrm{QoS}$ provisioning. As experiences have shown with Integrated Service and Differentiated Service defined by IETF, it is difficult over IP layer to ensure required throughput and other QoS parameters for individual data streams. End-to-end delay and jitter, on the other hand, mainly depend on performance of all switches along the transport path. A multi-layer switch will surely introduce more variable factors than a single layer one in addition to longer protocol processing delay. Therefore, one may ask: can we build a single-layer substrate?

Analysis has shown that main functions of the data link layer are framing and hop-by-hop error checking. It is very important to have hop-by-hop error checking and recovery mechanisms over high error rate links (e.g. subscriber loops in telephone system). Nevertheless they are no longer our concerns when error rates have been reduced to as low as $10^{-}$ ${ }^{11}$ to $10^{-12}$ over optical fibers or UTPs. Moreover, for various reasons, physical-layer framing has become very popular, such as those in PDH and ADSL. Incompatible frames at two adjacent layers can cause functional duplication and incur frame-length adaptation problem. All these considerations have suggested that an independent data link layer should no longer be justified with modern low error-rate communications. A single-layer substrate combining multiplexing and switching functions can be beneficial.

However, analysis of existing physical layer switching techniques has shown [16]:

A. Lambda or optical switching techniques lack downwards multiplexing mechanisms with fine granularity; hence they are unsuitable for single-layer substrate.
B. SDH is an excellent multiplexing technique over optical fibers but not a switching technique. Moreover, the need for adaptation between data link frames and SDH payload fields incurs extra processing. Finally, global synchronization as being used in SDH may be difficult to be realized in subnetworks, especially for ever higher frequencies.

C. MPLS has become a hot spot in the last ten years. Reference [12] provides a comprehensive discussion on MPLS with a negative conclusion: for those layer 2 subnetworks (ATM or FR) with good QoS insurance, MPLS only adds extra cost; while for those without such mechanism, it does not add any credits to QoS provisioning since it relies on lower layer mechanisms. In view MPLS adopts the patching strategy towards NGI; it is not a suitable candidate for backbone substrate.

D. Ethernet has made proud progress in the past and it tentimes its data rate every 5 to 7 years with $30 \%$ increase in cost. In view that $100 \mathrm{G}$ Ethernet standard is going to be published in year of 2010; it is a prominent candidate for backbone networks. However, its connectionless MAC service does not suitable for providing QoS insurable service hence needs improvement. Typical ongoing work with Ethernet includes PBT (Provider Backbone Transport) [18] and PBB (Provider Backbone Bridges) [19]. Despite pros and cons to them, one thing in common is that they are trying to enhance existing Ethernet, not to simplify it. In a matter of speaking, they are adopting the "add-on" strategy.

Since the year of 2000, SC-Netcom Lab has devoted its efforts to build a single-layer substrate and came out with a technique called EPFTS (Ethernet-oriented Physical-layer Frame Timeslot Switching) [20]. 


\section{EPFTS-BASED SUBSTRATE}

EPFTS stands for "Ethernet-oriented Physical Frame Timeslot Switching" intending to build a single-layer substrate for backbone subnetworks. The attribute "Ethernetoriented" here implies that it takes the most popular Ethernet MAC frames as the main "cargo" to be carried or "ferried" over the substrate since more than $95 \%$ of user premise networks are Ethernet-based. An Ethernet-oriented Physical Frame (EPF) consists of a 16-byte header and a 1982-byte "payload" hence makes it a fixed length of 2024 bytes (see Fig. 4).



Figure 4. Format of Ethernet-oriented Physical Frame

In Fig.4, the 1982-byte payload is catered for the "envelope frame" - the longest Ethernet MAC frame to avoid segmentation in encapsulation. Envelope frames are designed to allow inclusion of additional prefixes and suffixes required by higher layer encapsulation protocols such as those defined by the IEEE 802.1 working group (such as Provider Bridges and MAC Security), ITU-T or IETF (such as MPLS) [20]. However, for EPF they are merely "cargoes" and will be "ferried" in EPF-based substrate without the need for understanding their meanings. Therefore, an EPF is the basic data unit in EPFTS platform and plays the "ferrying" functions for higher-layer data. The time for transmitting or switching an EPF is referred to as a timeslot. Transmission and switching of EPFs over a lambda channel or within a switch fabric in a node work in an asynchronous mode, i.e. gaps between EPFs or timeslots are variable. Consequently, EPFTS over DWDM provides an EPF-based multi-granular multiplexing and switching capability with embedded QoS mechanisms and Operation \& Administration (O\&A) functions. Fig.5 illustrates the format of 16-yte header, which supports a multi-cast operation.

\begin{tabular}{|c|c|c|c|c|c|c|c|}
\hline \multicolumn{4}{|c|}{$\longleftarrow 1^{\text {ti }} 8$-byte block in EPF Header $\longrightarrow$} & \multicolumn{4}{|c|}{$\downarrow \quad 2^{\text {nd }} 8$-bvte block in EPF Header $\longrightarrow$} \\
\hline 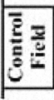 & \begin{tabular}{c|} 
Reserved for \\
Operation \& \\
Administration
\end{tabular} & \multicolumn{2}{|c|}{$\begin{array}{l}\left(\mathrm{SFS}_{1}\right) \text { Step-Forward } \\
\text { Switching Field } 1\end{array}$} & \multicolumn{2}{|c|}{$\begin{array}{c}\text { (SFS,) Step-Forward } \\
\text { Switching Field } 2\end{array}$} & \multicolumn{2}{|c|}{\begin{tabular}{|c|} 
(SFS 3 ) Step-Forward \\
Switching Field 3
\end{tabular}} \\
\hline \\
\hline 产产 & 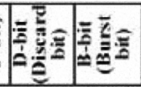 & $\begin{array}{l}\text { E-bit } \\
\text { (End } \\
\text { bit) }\end{array}$ & $\begin{array}{l}\text { Priorit } \\
\text { (000: the his } \\
\text { the lor }\end{array}$ & $\begin{array}{l}\text { ty bits } \\
\text { ighest, } 111: \\
\text { west) }\end{array}$ & $=$ & 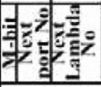 & $\begin{array}{c}\begin{array}{c}\text { Virtual } \\
\text { Line } \\
\text { Identifler }\end{array} \\
\end{array}$ \\
\hline
\end{tabular}

Figure 5. Format of EPF header supporting multi-casting

As shown in Fig.5, the 16-byte header is further divided into three parts: 1-byte control field, 3 bytes reserved field for O\&A (Operation \& Administration), and Step-forward Switching fields, 4 bytes each.

The $1^{\text {st }}$ bit in Control field, i.e. the S-bit, signifies that an EPF is carrying "cargo" for S\&M-platform (when equals to "1") or for U-platform (when equals to "0"). When the D-bit is set to " 1 ", it implies that this EPF is liable to be discarded in congestion condition. The B-bit (Burst bit) provides a mechanism for bursts such as defined in the 802.3 group, to maintain integrity of multiple Ethernet MAC frames. The Ebit, on the other hand, provides a simple way to disconnect a connection. Finally the three priority bits are used to indicate switching priority for different types of traffic [17].

EPFTS innovatively takes the advantage of connectionoriented service by utilizing information available during connection establishment phase to enable "Step-forward Switching", with which an output EPF of the current node carries the output port and lambda numbers and input indexes of the downstream node. Therefore, any incoming EPF can tell a node without the need for looking up a switching table which output port(s)/lambda(s) this EPF should be switched to. Three SFS fields can support up to 3 multi-cast branches at any node and a " 1 " in M-bit in each SFS field signifies that the EPF should be copied to more output branch.

It should be noted that the VLI (Virtual Line Identifier) implies that EPFTS provides a connection-oriented switching service at the physical layer. They can be either a Permanent Virtual Line (PVL) service or a Switched Virtual line (SVL) service. To cater for different QoS requirements, a virtual line can be dedicated to a single time-critical data stream (similar to Integrated Service), or multiple data streams with similar QoS (similar to Differentiated Service), or for large amount of data streams with "best effort service" requirement.

It is obvious that a multi-concatenated VLI actually forms an End-to-End path in EPFTS domain and can be viewed as tunnels at the Physical layer. Therefore, the ferrying service provided by EPFTS can be seen as physical layer tunneling service with Integrated Service or Differentiated Service flavor.

EPFTS in optical transmission adopts the 64B66B encoding and scrambling technique, which has been widely used in Ethernet technologies at rates of $10 \mathrm{Gbps}$ and above [21, 22]. With 64B66B encoding, 64-bit data blocks are scrambled to avoid continuous " 1 " or " 0 " and two extra un-scramble bits are added in front of the 64-bit block to differentiate "user data" from "control data". This can easily be mapped onto the "S-bit" in EPF when entering into EPFTS domain and consequently, only 64 bits need to be switched within a node and the two extra bits will be re-attached to the blocks for output according to the value of "S-bit".

2024-byte frame length against 16-byte header, the overhead for EPFTS is lower than $0.1 \%$, which is much less than that of ATM cell (10\%).

\section{SUPA AND SUPANET}

With the out-of-band signaling concept, we have, by separation of the U-platform from S\&M-platform, simplified the U-platform to a single layer with EPFTS while keeping the multi-layer protocol stack of Internet in the S\&Mplatform with necessary enhancement. This actually results in a "Single-layer User-data switching Platform Architecture (SUPA) [6]. In a broad sense, any network, of which U- 
platform involves only the physical layer, can be seen as a SUPANET. But in this paper, the term SUPA is devoted to an EPFTS-based single U-platform network architecture.

Research and development on SUPANET at SC-Lab are divided into three phases:

A. Phase 1: preliminary study on SUPA focusing on EPFTS in U-platform and remaining existing Internet protocol stacks in $S \& M$-platform with necessary enhancement.

B. Phase 2: EPFTS based substrate experiment for both $U$ platform and $S \& M$-platform with further refinement of SUPA.
C. Phase 3: further simplification to S\&M-platform with the 3-layer End-system model and exploration of demarcating Network Applications from Network Application Support Service aiming for a future network model as illustrated in Fig. 3.

So far we have finished with the task for phase 1 and entered phase 2 researches and experiment. Some preliminary work for phase 3, such as examine common application support functions, have also started. Fig. 6 illustrates the architecture developed in phase 1:

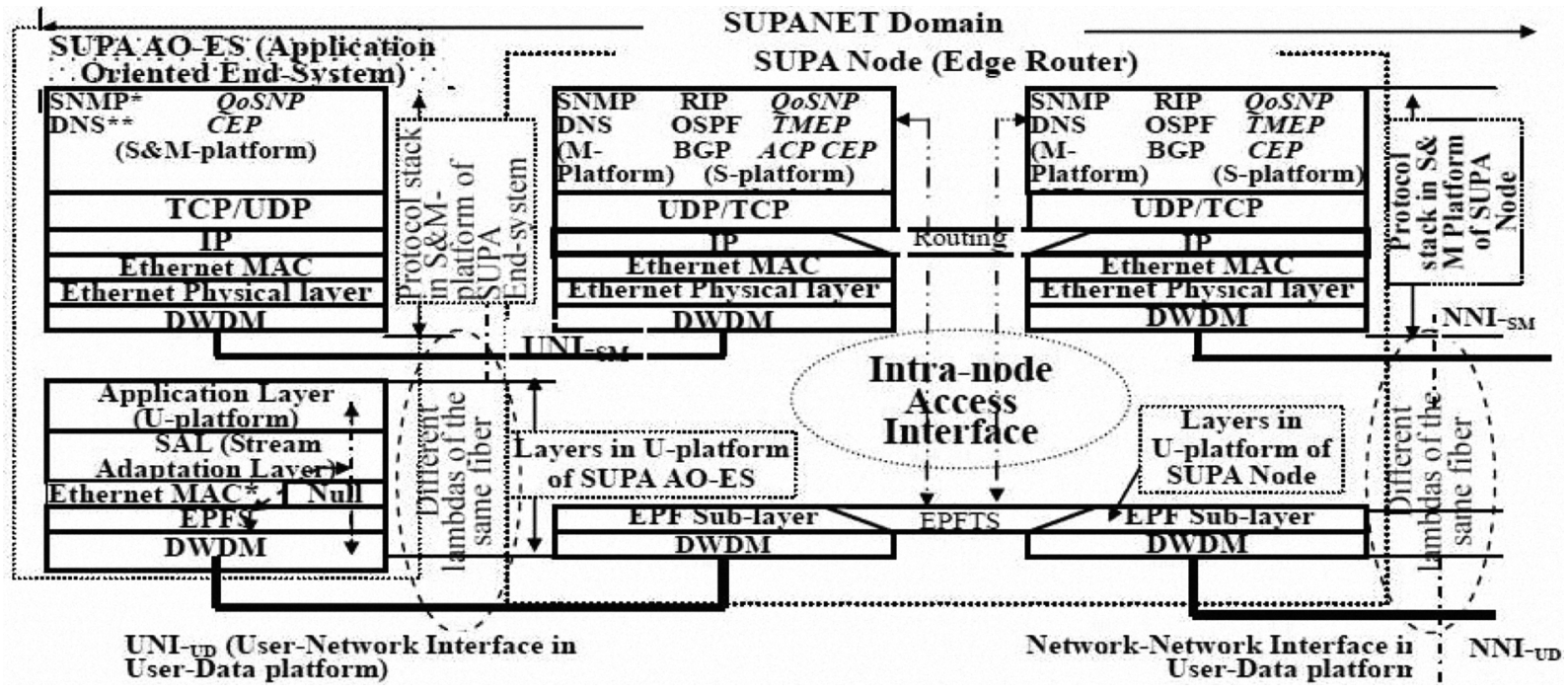

Figure 6. SUPA Phase One Model and protocol stacks in U-platform and S\&M-platform

As shown in Fig.6, the phase one model is not only a conceptual model, but also an implementation model for SUPA node, which represents an enhanced router closely coupled with an EPFTS switching devices via the Intra-node Access Interface (IAI). The SUPA AO-ES (Application Oriented End-System) in Fig. 6 represents either a real SUPA host or a half-gateway at SUPANET side. The S\&M-platform of an SUPA AO-ES supports all the Internet protocol stacks necessary for Internet operation with a few enhancement protocols such as QoSNP (QoS Negotiation Protocol) and CEP (Call Ending Protocol). While in its U-platform, user application data can be segmented and adapted via SAL (Stream Adaptation Layer) either directly to EPF or indirectly through Ethernet MAC frame.

The Edge Router in Fig. 6 represents two types of half switch: one interfaces with AO-ES via UNI (User-Network Interface and the other interfaces non-edge routers via NNI (Network-Network Interface). In S\&M-platform, the edge side differs from the other side by having Call Admission Control (CAC). In the U-platform, User-data Admission Con- trol function is implemented at the edge side to manage input traffic rate according to QoS contract and network congestion condition.

One of the important functions in S\&M-platform is to negotiate QoS for individual Virtual Line traffic among two AO-ESs and routers to find an end-to-end path between data source and destination and map the path in U-platform as concatenated VLs. As mentioned in section VI, all the information needed for Step-Forward Switching (SFS) are stored in U-platform of MIB (Management Information Base) in relevant routers along the path for up-dating.

During phase one research and development, we have established a set of mechanism to ensure required QoS for different traffic spanning from QoSNP, Two-step routing (port and lambda level), Quota assignment and dynamic adjustment, Traffic Engineering, in S\&M-platform and those over EPFTS platform. Reference [23] provides a comprehensive discussion on these issues. 


\section{CONCLUSION AND FUTURE WORKS}

This paper has provided a brief discussion on new requirements of network applications, challenges faced with Internet and our solutions. This paper promotes a BSF-OES development strategy for NGI and a future NGI model, which are further exemplified by SUPA and its core switching techniques EPFTS. Our phase one development has convinced us that our research work is in the right track supported by a large amount of experiments and simulations. However, we are a little skeptical about merely relying on simulations and are currently building a hardware-based experimental EPFTS platform to further validate our results. We are aware that development of NGI is a long-term task and requires international cooperation as well as competition with different approaches. In our second and third phases, there are a lot of problems to be solved such as security issues for future networks, demarcation of network support functions, real network applications, and etc.

\section{ACKNOWLEDGEMENT}

The work presented in this paper has been supported by two consecutive grants from CNSF (with grant numbers 60372065 and 60773102 respectively). The authors wish to thank the financial support from CNSF.

\section{REFERENCES}

[1] Steven M. Bellovin, David D. Clark, Adrian Perrig, and Dawn Song, A Clean-Slate Design for the Next-Generation Secure Internet (GENI Design Documents, GDD-05-05), NSF Workshop Report, July 2005.

[2] ISO/OSI, Basic Reference Model for Open System Interconnection (OSI/RM), IS7498, October 1984.

[3] I. Foster, and et al, The Open Grid Services Architecture - GWD-R (draft-ggf-ogsa-ogsa-08), September 21, 2003.

[4] ZENG Hua-shen, LI ji, FENG Lin, and GONG Xue, "On computer networks and grid service", Computer Applicaions (in Chinese), vol.25, no.7, pp. 1475-1478, July 2005.

[5] Cloud computing, http://en.wikipedia.org/wiki/Cloud_computing

[6] Huaxin Zeng, Jun Dou and Dengyuan Xu, "Single physical layer Uplane Architecture (SUPA) for Next Generation Internet", Comprehensive Report on VoIP and enhanced IP Communications Services, IEC Publications, 2004, pp. 197-227.

[7] OASIS Standard, Reference Model for Service Oriented Architecture 1.0, October 12, 2006, http://docs.oasis-open.org/soa-rm/v1.0/.

[8] Overlay network, http://en.wikipedia.org/wiki/Overlay_network

[9] Rüdiger Schollmeier, "A Definition of Peer-to-Peer Networking for the Classification of Peer-to-Peer Architectures and Applications", Proceedings of the First International Conference on Peer-to-Peer Computing, IEEE, Augest 27-29, 2001, pp.101-102.

[10] J. Postel and J. Reynolds, File Transfer Protocol, RFC0959, IETF, October 1985.

[11] R. Braden, Ed., L. Zhang, S. Berson, S. Herzog, and S. Jamin, Resource ReSerVation Protocol (RSVP) -- Version 1 Functional Specification RFC2205, IETF, September 1997.

[12] Huaxin Zeng, Jun DOU, and Dengyuan XU, "Replace MPLS with EPFTS to build a SUPANET", Proceedings of 2005 IEEE International Workshop on High Performance Switching and Routing (HPSR'05), Hong Kong, May 2005, pp.39-43.

[13] Huaxin Zeng, Yu Gao and Yu Xia, "On NGN architecture and evolution strategy", Proceedings of the first ITU-T Kaleidoscope Academic
Conference, Innovations in NGN: Future Network and Services, Geneva, May 12-13, 2008, pp. 337-342.

[14] Clark D, Braden R, Falk A, and Pingali V, "FARA: Reorganizing the addressing architecture", Proceedings of the ACM SIGCOMM, ACM Press, 2003, pp. 313-321.

[15] Zeng Jiazhi, Xu Jie, Wu Yue, Li Yichao and Xu Neng, "Service Unit based Network Architecture," Proceedings of PACAT03, IEEE press, Aug, 2003, pp. 12-16.

[16] Huaxin Zeng, Dengyuan Xu, and Jun Dou, "Promotion of Physical Frame Timeslot Switching (PFTS) over DWDM", Annual Review of Communications, vol.57, IEC Publications, 2004, pp. 809-826.

[17] XU Dengyuan, ZENG Huaxin, Li Ji, GUO Zirong, "Physical Frame Timeslot Switching (PFTS) in the Single User-Plane Architecture Network (SUPANET)", Proceedings of 5th International Conference on Parallel and Distributed Computing, Applications and Technologies (PDCAT2004), Singapore, December 2004, Lecture Notes in Computer Science (LNCS 3320), Springer, pp.383-395.

[18] IEEE 802.1Qay, Provider Backbone Bridge Traffic Engineering, http://www.ieee802.org/1/pages/802.1ay.html

[19] IEEE 802.1ah, Provider Backbone Bridges, Http://www.ieee802.org/1/ pages/802.1ah.html

[20] IEEE Std 802.3as, Amendment 3: Frame format extensions, November 13, 2006

[21] IEEE Std 802.3, Part 3: Carrier sense multiple access with collision detection (CSMA/CD) access method and physical layer specifications, December 9,2005

[22] http://grouper.ieee.org/groups/802/3/ba/BaselineSummary_0908.pdf, IEEE P802.3ba 40Gbs and 100Gbs Ethernet Task Force Meeting, May 13,2008

[23] Jun DOU, Huaxin Zeng, and Haiying Wang, "Single User-Plane Architecture and its QoS provisioning mechanisms in Signaling and Management (S\&M) Planes", Proceedings of 5th International Conference on Parallel and Distributed Computing, Applications and Technologies (PDCAT2004), Singapore, December 2004, Lecture Notes in Computer Science (LNCS 3320), Springer, pp. 429-440. 